\title{
A FIXED POINT THEOREM FOR SET VALUED MAPPINGS ${ }^{1}$
}

\author{
BY JACK T. MARKIN
}

Communicated by C. B. Morrey, Jr., February 5, 1968

Let $H$ be a real Hilbert space with closed unit ball $B$ and let $K(H)$ denote the family of nonempty compact convex subsets of $H$ supplied with the Hausdorff metric $D$ generated by the norm of $H$. A mapping $\phi: H \rightarrow K(H)$ is contractive if for any pair $x, y \in H, D(\phi(x), \phi(y))$ $\leqq D(x, y)$. If $x \in \phi(x)$, then $x$ is a fixed point of $\phi$.

In this paper we shall prove the following fixed point theorem for set valued contractions, which is an extension of a theorem of Browder [1].

THEOREM 1. Let $\phi: H \rightarrow K(H)$ be a contractive mapping such that $\phi(x) \subset B$ for every $x \in B$. Then $\phi$ has a fixed point in $B$.

The proof relies on a generalization of the concept of monotone mappings of $H$ into $H$ to mappings of $H$ into $K(H)$, and also depends on Theorem 2 which we state without proof.

Theorem 2. Assume that $X$ is a complete bounded metric space and that $\phi$ maps $X$ into the family of nonempty closed subsets of $X$. If there is a $k \in[0,1)$ such that for any pair $x, y \in X, D(\phi(x), \phi(y)) \leqq k D(x, y)$, then $\phi$ has a fixed point. (Here $D$ is the Hausdorff metric generated by the metric of $X$.)

A mapping $G$ of $H$ into the family of nonempty subsets of $H$ is monotone if given $u, v \in H$ and $\bar{u} \in G(u)$ there is a $\bar{v} \in G(v)$ such that $(\bar{u}-\bar{v}, u-v) \geqq 0$.

LEMma 1. Let $G: H \rightarrow K(H)$ be a continuous monotone map, and assume that for some pair $v, \bar{v} \in H$ and every $u \in H$ there is a $\bar{u} \in G(u)$ such that $(\bar{v}-\bar{u}, v-u) \geqq 0$. Then, $\bar{v} \in G(v)$.

Proof. Suppose $\bar{v} \notin G(v)$. By weak compactness, there is a $w \in H$ such that $(\bar{v}, w)<(x, w)$ for every $x \in G(v)$. Let $v_{n}=v-(1 / n) w$; since $G$ is monotone there exists a $\bar{v}_{n} \in G\left(v_{n}\right)$ such that $\left(\bar{v}-\bar{v}_{n}, v-v_{n}\right) \geqq 0$ for all $n$. Therefore $(\bar{v}, w) \geqq\left(\bar{v}_{n}, w\right)$. Since $D\left(G\left(v_{n}\right), G(v)\right)$ tends to 0 as $n \rightarrow \infty$ by continuity, we may assume that $\left\{\bar{v}_{n}\right\}$ tends weakly to a point $\bar{x}$ and that there is a sequence $\left\{z_{n}\right\}, z_{n} \in G(v)$, such that

${ }^{1}$ This work was supported by the U. S. Army Research Office-Durham, contract DA-31-124-ARO-D-265. 
$\lim D\left(\bar{v}_{n}, z_{n}\right)=0$ and $\left\{z_{n}\right\}$ converges weakly to a point $z_{0} \in G(v)$. We assert that $\bar{x} \in G(v)$ so that $(\bar{v}, w) \geqq(\bar{x}, w)$, which is absurd. Indeed, if $\bar{x} \notin G(v)$ there is a $\bar{z} \in H$ such that $(\bar{x}, \bar{z})<\left(z_{0}, \bar{z}\right)$ so that

$$
0<\left(z_{0}-\bar{x}, \bar{z}\right)=\left(z_{0}-z_{n}, \bar{z}\right)+\left(z_{n}-\bar{v}_{n}, \bar{z}\right)+\left(\bar{v}_{n}-\bar{x}, \bar{z}\right),
$$

which is absurd (because the right side converges to 0 as $n \rightarrow \infty$ ).

Lemma 2. If $G: H \rightarrow K(H)$ is a continuous monotone mapping then $G(B)$ is closed in the norm topology.

Proof. If $v_{0}$ is a limit point of $G(B)$ there is a sequence $\left\{\bar{u}_{j}\right\}$ in $G(B)$ such that $\bar{u}_{j} \in G\left(u_{j}\right), \bar{u}_{j} \rightarrow v_{0}$ and $u_{j} \in B$. Since $B$ is weakly compact we may assume that $u_{j}$ converges weakly to $u_{0} \in B$.

If for every $u \in H$ there is a $\bar{u} \in G(u)$ such that $\left(\bar{u}-v_{0}, u-u_{0}\right) \geqq 0$, then Lemma 1 implies that $v_{0} \in G\left(u_{0}\right)$ and the proof is complete. If not, there is a $v \in H$ and an $\epsilon>0$ such that

$$
\left(\bar{v}-v_{0}, v-u_{0}\right) \leqq-\epsilon<0
$$

for all $\bar{v} \in G(v)$. We shall show that this leads to a contradiction.

Since $G$ is monotone there is a $\bar{v}_{j} \in G(v)$ satisfying $\left(\bar{v}_{j}-\bar{u}_{j}, v-u_{j}\right) \geqq 0$ for each positive integer $j$. By the compactness of $G(v)$ we may assume that $\bar{v}_{j} \rightarrow w \in G(v)$. Therefore, $\left(\bar{v}_{j}-\bar{u}_{j}, v-u_{j}\right) \rightarrow\left(w-v_{0}, v-u_{0}\right) \geqq 0$, which contradicts (1).

Given any $u, v \in H$ and $\bar{u} \in \phi(u)$ the compactness of $\phi(v)$ guarantees a point $\bar{v} \in \phi(v)$ such that $D(\bar{u}, \bar{v}) \leqq D(\phi(u), \phi(v))$. Therefore, $((u-\bar{u})-(v-\bar{v}), u-v) \geqq(D(u, v))^{2}-D(\bar{u}, \bar{v}) \cdot D(u, v) \geqq 0$, and hence $I-\phi$ is a monotone mapping. Clearly, $I-\phi$ is continuous as a mapping of $H$ into $K(H)$.

If we show that 0 is in the closure of $(I-\phi)(B)$, then Theorem 1 will follow from the lemmas.

Let $\left\{k_{i}\right\}$ be a sequence in $(0,1)$ which converges to 1 . For each $i$, $k_{i} \phi$ maps $B$ into the family of nonempty closed convex subsets of $B$, and satisfies the hypotheses of Theorem 2. Therefore, for each $i$ there is a fixed point $u_{k_{i}} \in k_{i} \phi\left(u_{k_{i}}\right)$. Clearly, $u_{k_{i}}=k_{i} v_{k_{i}}$ for some $v_{k_{i}} \in \phi\left(u_{k_{i}}\right)$ and hence

$$
\inf _{y \in \phi\left(u_{k_{i}}\right)} D\left(u_{k_{i}}, y\right) \leqq D\left(u_{k_{i}}, v_{k_{i}}\right) \leqq 1-k_{i} \rightarrow 0 \text { as } i \rightarrow \infty .
$$

\section{REFERENCE}

1. F. E. Browder, Fixed point theorems for non-compact mappings in Hilbert space, Proc. Nat. Acad. Sci. U.S.A. 53 (1965), 1272-1276.

University of Southern California 